\title{
A Review of Physical Human Activity Recognition Chain Using Sensors
}

\author{
Omar AlShorman ${ }^{1}$, Buthaynah AlShorman², and Mahmoud S. Masadeh ${ }^{3}$ \\ ${ }^{1}$ Electrical Engineering Department, Faculty of Engineering and AlShrouk Trading Company, Najran University, Najran, \\ Saudi Arabia \\ ${ }^{2}$ South Alternative Center for Physiotherapy and Rehabilitation, Najran, Saudi Arabia \\ ${ }^{3}$ Computer Engineering Department, Yarmouk University, Irbid, Jordan
}

\section{Article Info}

Article history:

Received Mar 21, 2020

Revised Aug 31, 2020

Accepted Oct 3, 2020

\section{Keywords:}

Healthcare Monitoring

Human Activity Recognition

Internet of Medical Things

Machine Learning

Sensors

\begin{abstract}
In the era of Internet of Medical Things (IoMT), healthcare monitoring has gained a vital role nowadays. Moreover, improving lifestyle, encouraging healthy behaviours, and decreasing the chronic diseases are urgently required. However, tracking and monitoring critical cases/conditions of elderly and patients is a great challenge. Healthcare services for those people are crucial in order to achieve high safety consideration. Physical human activity recognition using wearable devices is used to monitor and recognize human activities for elderly and patient. The main aim of this review study is to highlight the human activity recognition chain, which includes, sensing technologies, preprocessing and segmentation, feature extractions methods, and classification techniques. Challenges and future trends are also highlighted.
\end{abstract}

Copyright $@ 2020$ Institute of Advanced Engineering and Science. All rights reserved.

\section{Corresponding Author:}

Omar AlShorman

Electrical Engineering Department, Faculty of Engineering and AlShrouk Trading Company, Najran

University, Najran, Saudi Arabia

Email: omar2007_ahu@yahoo.com

\section{INTRODUCTION}

Physical activity (PA) and sports are highly associated with health [1-3]. Hazardously, physical inactivity is considered as the fourth risk factor for mortality in the world [4]. Scientific evidences consider physical activity and sports are key elements of health and wellbeing [5, 6]. Moreover, regular PA has physical and mental health benefits such as [7-10]:

- Decreasing the risk of chronic diseases such as diabetes, cardiovascular, stroke, depression, anxiety, high blood pressure, heart disease, many types of cancer, hypercholesterolemia, and arthritis.

- Controling the weight and obesity.

- Enhancing cognitive performance.

- Improving the mood and solving sleep difficulties.

- Building healthy muscle, bones, and joints.

Nowadays, improving lifestyle, encouraging healthy behaviors, and decreasing the chronic diseases are urgently required [11, 12]. Importantly, the use of advanced technology and computer capabilities in healthcare industries [13] has gained a vital role of our daily lives [14, 15]. However, tracking and monitoring elderly and patients are challenges [16]. Healthcare services for those people are vital in order to achieve high safety living [17-19]. Wearable devices are now highly used for human activity recognition. They are used as a predictor for any abnormal health cases [20, 21]. Figure 1 shows human activity monitoring using wearable sensors. 
The main aim of this review study is to present the importance of modern technologies for improving the healthy behavior and lifestyle of the humans. Moreover, physical human activity recognition chain using wearable sensors is highlighted.

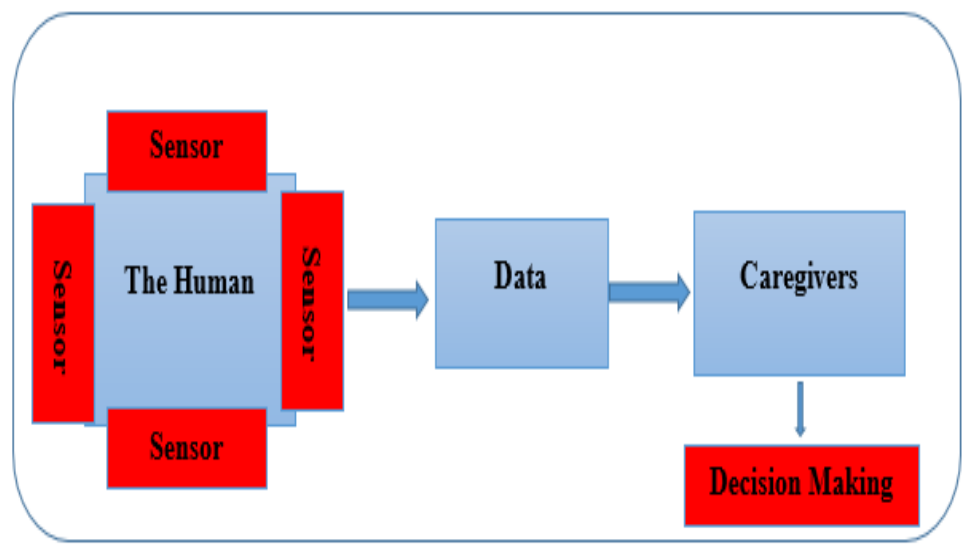

Figure 1. Human activity monitoring using wearable sensors

\section{HUMAN ACTIVITY RECOGNITION}

The analysis of human activity data is crucial [22]. However, data obtained by sensors can be used as a predictor of health status. Basically, the recognition of activities can be divided into two categories, external sensors and wearable sensors [23]. A main example of external sensors is smart homes [24, 25] which have the ability to recognize complex activities [26] such as preparing a meal and answering a phone call. Nowadays, in computer vision and machine learning fields, human activity recognition using wearable sensors has become one of the state-of-the-art research areas, where Figure 2 shows human activity recognition chain [27].

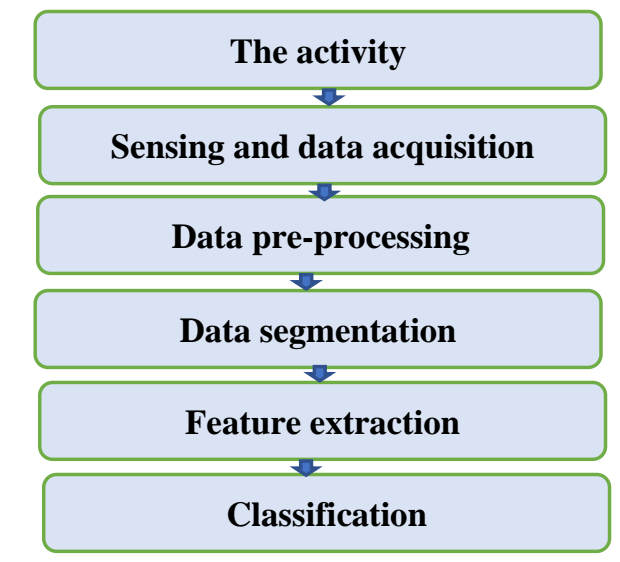

Figure 2. Human activity recognition chain.

Initially, to make the data suitable for analysis, obtained sensor's data are preprocessed [28] before it goes through the rest of the chain. As example, signals have to be filtered firstly. In order to remove high frequency components, low pass filters are used. In addition to filtering technique, data conversion, calibration, normalization, and cleaning may be used in this stage. The main aim of the segmentation stage [29] is to seek the segments, which contain a valuable data about activities, and to reduce the amount of processed data. Sliding window techniques are the common example of the segmentation stage [30]. Feature extraction stage [31-34] is used to find a high resolution of data representation for each segment. Extracted features can be divided into four main categories: time domain, frequency domain, learning techniques, and other techniques. Tables 1 and 2 summarize the time and frequency domain-based techniques, respectively. Table 1 summarizes time domain based techniques. Moreover, Table 2 summarizes frequency domain based techniques, while Table 3 summarizes intelligent learning based techniques and Table 4 summarizes intelligent learning based techniques. 
Table 1. Physical human activity recognition feature extraction and selection techniques based on time domain techniques

\begin{tabular}{lc}
\hline \multicolumn{1}{c}{ The technique } & References \\
\hline Statistical values: mean, variance, skewness & {$[35]$} \\
and kurtosis & {$[36]$} \\
Maximum and minimum values & {$[37]$} \\
Fourier transform & {$[38]$} \\
Wavelet transform & {$[39]$} \\
Entropy & {$[40]$} \\
Mean Absolute Deviation (MAD) & {$[41]$} \\
Signal magnitude area & {$[42]$} \\
Zero crossing rate & {$[43]$} \\
Interquartile range & {$[44]$} \\
Hidden conditional random fields & \\
\hline
\end{tabular}

Table 2. Physical human activity recognition feature extraction and selection techniques based on frequency domain techniques

\begin{tabular}{lc}
\hline \multicolumn{1}{c}{ The technique } & References \\
\hline Discrete Fourier Transform (DFT) & {$[45]$} \\
Discrete Cosine Transform (DCT) & {$[46]$} \\
Spectral centroid & {$[47]$} \\
Principal frequency & {$[48]$} \\
Frequency domain entropy & {$[49]$} \\
Maximum frequency & {$[50]$} \\
Fast Fourier Transform (FFT) & {$[51]$} \\
FFT energy & {$[52]$} \\
FFT mean and standard deviation & {$[53]$} \\
Mel-Frequency Cepstrum & {$[54]$} \\
Perceptual Linear Predictive Cepstrum & {$[55]$} \\
\hline
\end{tabular}

Table 3. Physical human activity recognition feature extraction and selection techniques based on intelligent

\begin{tabular}{lc}
\multicolumn{2}{c}{ learning } \\
\hline \multicolumn{2}{c}{ The technique } \\
\hline Artificial Neural Networks & {$[56,57]$} \\
Hidden Markov Fields (HMF) & {$[58,59]$} \\
Dynamic Bayesian Networks (DBN) & {$[60]$} \\
Codebook approach (CB) & {$[61]$} \\
Convolutional Neural Network (CNN) & {$[62]$} \\
Hand-crafted features (HC) & {$[63]$} \\
Multi-Layer-Perceptron (MLP) & {$[64]$} \\
Long Short-Term Memory network (LSTM) & {$[65]$} \\
Autoencoder (AE) & {$[66]$} \\
\hline
\end{tabular}

Table 4. Physical human activity recognition feature extraction and selection techniques based on other

\begin{tabular}{lc}
\multicolumn{2}{c}{ learning } \\
\hline \multicolumn{1}{c}{ The technique } & References \\
\hline Autoregresive Model (AR) & {$[67]$} \\
Multi-class Linear Discriminant Analysis (MLDA) & {$[68,69]$} \\
Principal Components Analysis (PCA) & {$[70]$} \\
HAAR filters & {$[71]$} \\
Shape analysis & {$[72]$} \\
Bag-of-Words & {$[73]$} \\
Linearly Dependent Concept & {$[74]$} \\
\hline
\end{tabular}

In the classification stage, the classifier (classification algorithm/technique) uses the extracted features to differentiate between different human activities [75, 76]. Table 5 summarizes different classification algorithms. 
Table 5. Classifiers used for physical human activity recognition

\begin{tabular}{|c|c|}
\hline Classification technique & References \\
\hline Autoregresive Model (AR) & {$[77,78]$} \\
\hline Linear regression & {$[79,80]$} \\
\hline Logistic regression & {$[81,82]$} \\
\hline Markov Models & {$[83,84]$} \\
\hline Random Forest & {$[85,86]$} \\
\hline Boosting and Bagging & {$[87,88]$} \\
\hline Sequential Minimal Optimization & [89-91] \\
\hline Fuzzy Logic & [92-94] \\
\hline Relevance vector machines & {$[95,96]$} \\
\hline Support Vector Machines & {$[97,98]$} \\
\hline Least Squares Support Vector Machines & {$[99,100]$} \\
\hline Adaptive minimum distance & {$[101,102]$} \\
\hline Gaussian Mixture Model (GMM) & {$[103,104]$} \\
\hline Naive Bayes & {$[105,106]$} \\
\hline Gaussian naive bayes & {$[107,108]$} \\
\hline Bernoulli naive bayes & {$[109,110]$} \\
\hline Multinomial naive bayes & {$[111,112]$} \\
\hline Bayesian Networks & {$[85,113,114]$} \\
\hline K-nearest neighbors & {$[115,116]$} \\
\hline Zero rule & {$[116,117]$} \\
\hline One rule & {$[103,118]$} \\
\hline Decision tree & {$[119,120]$} \\
\hline Fisher's linear discriminant & {$[121,122]$} \\
\hline Decision stump & {$[123,124]$} \\
\hline Stochastic gradient descent & {$[125,126]$} \\
\hline Linear discriminant analysis & {$[127,128]$} \\
\hline Quadratic discriminant analysis & [129-131] \\
\hline Boosted tree & {$[132,133]$} \\
\hline Conditional Random Fields & {$[134,135]$} \\
\hline Skip Chain & {$[136,137]$} \\
\hline Codebook & {$[138,139]$} \\
\hline Linear Predictive Coding & {$[140,141]$} \\
\hline Neural Network: Multilayer Perceptron & {$[142,143]$} \\
\hline Neural Network: Basic radial function & [144-146] \\
\hline Multilayer Neural Networks & [144-146] \\
\hline Deep Learning: Convolutional Neural Networks (CNNs) & {$[139,147-153]$} \\
\hline Deep Learning : Deep Belief Networks (DBNs) & [154-156] \\
\hline Deep Learning: Autoencoder & [157-161] \\
\hline Deep Learning: Sparse Coding & [162-164] \\
\hline Deep Learning: Recurrent Neural Network (RNN) & {$[165-168]$} \\
\hline Deep Learning: Boltzmann machine & [169-172] \\
\hline Deep Learning: Feedforward Neural Network (FNN) & [173-175] \\
\hline
\end{tabular}

\section{WEARABLES AND SENSING TECHNOLOGY}

Currently, wearable devices and sensors [176-180] (such as pulse monitors, mobile phones, smart watches, and smart glasses) are used in many modern applications such as industry, medical field, and security [181-183]. In the medical applications, the main purpose of these devices and sensors is to obtain a reliable data to use them in monitoring/tracking people's activities and behaviors [184]. However, signs of humans' body such as brain signals, blood pressure, temperature, heart rate, motion, spinal posture, sweat rate, respiration rate and glucose level can be monitored [185]. Moreover, with the help of wearable devices and sensors[186], healthcare providers can continuously and remotely monitor all signs and activities [187190]. As an example of remote activity monitoring, sensors can be used to track subject's motion and unexpected activities, such as fall detection [191].

In the PA and sports, activity recognition [192-194] can be used to obtain physical activities such as running, driving, jumping, swimming, dancing, playing sports, walking, lying, standing, sitting, hiking, and 
jogging $[195,196]$. Several studies have applied activity recognition for patients, physically or mentally disabled people, children, and elderly [197]. Thus, wearable computing and sensing technology will positively enhance health and medical technology [198]. This leads to reduce medical cost, cure at home, redefine the doctor-patient relationship, and enhance medical services [199, 200]. Several types of sensors are developed and applied for the monitoring of activities and physiological parameters [201]. Table 6 summarizes available market sensors used in physical human activity monitoring [34, 202, 203].

Table 6. Available market sensors used in physical human activity monitoring

\begin{tabular}{ll}
\hline \multicolumn{1}{c}{ Sensor } & \multicolumn{1}{c}{ Application } \\
\hline Piezoresistive Sensor [204] & Force or pressure measurement \\
Sweat rate sensor [205] & Sweat measurement \\
Inertial sensors [206, 207] & Linear and angular accelerations \\
& measurements \\
Accelerometers [208] & Acceleration measurement \\
Shoe monitor sensor [209] & Locomotion measurement \\
ElectroCardiogram (ECG) sensors [210] & Rate and regularity of the heart beats \\
& measurement \\
Body temperature sensor [211] & Skin temperature measurement \\
Blood pressure sensor [212] & Blood pressure measurement \\
Pulse oximetry sensor [213] & Oxygen saturation level in blood measurement \\
Glucose sensor [214] & Glucose rate measurement \\
Smart phones [215, 216] & Several messurments \\
Cameras [217] & Recognition of activities and gestures from \\
GPS [218, 219] & video sequences \\
Spirometer, Electrooculography (EOG), and galvanic skin [220] & Human's movment \\
Electroencephalogram (EEG) [221, 222] & Physiological sensor \\
Magnetic field sensor [223] & Brain signals \\
\hline
\end{tabular}

\section{CHALLENGES AND FUTURE TRENDS}

This section summarizes human activity recognition challenges and future trends [76, 224-239]:

- Diversity of physical activities: definition of the activities and their specific characteristics is challenging task.

- Activity variation: certain activity may lead to multiple different styles of human motion.

- Outdoor/ uncontrolled environment: background noise may affect human activity recognition algorithms.

- Data collection and unavailability of big datasets.: intelligent algorithms for human activity recognition needs sufficient and big training data. Most available datasets are laboratory datasets.

- Feature extraction: knowledge expert- driven feature extraction methodes have to be extensivly discussed. However, time and frequency methodes do not have the ability to deal with dynamic nature of human activities.

- Computational time: new feature extraction processes need more computational time.

- Performance and accuracy: multiple sensors or multiple classifiers have to be used

- Intra-class variability and inter-class similarities: Humans act differently.

- Performance evaluation: false negative state must be reducing.

- Complex activities: complex and multitasking activities are diffecult to recognize.

- The NULL Class: required activities may interfere with activities that have similar behaviour/patterns but that are irrelevant to the scope.

- Class imbalance: the number of instances of one class far exceeds the other.

- Flexibility: the HAR system must be flexible to add new users without needing to re-train the system.

- Privacy and security: threats maybe occure due to highly sensitive information.

- Safety: touchable batteries maybe dangerous.

- Wearable sensors placement: how and where the wearable sensors can placed and attached to related locations in the body.

Design of wearable sensors: the sensor must be easy and comfortable for the users. 
- $\quad$ Single sensor modalities: information fusion stratigies have to discussed more.

- Energy efficiency: online continous sensing is energy consuming.

- The size: size of the sensor must be reduced.

- Obtrusiveness: human does not able to wear many sensors.

- Ergonomics: wearable devices must be comfortable for the users.

- $\quad$ Sealing: wearable devices must be protected from water and sweat.

- $\quad$ Noise: various noise levels for the same modality may occur.

\section{CONCLUSION}

In the IoMT era, analysis of human activity data is vital and represents the future of healthcare industry. Signs of humans' body such as brain signals, blood pressure, temperature, heart rate, motion, spinal posture, sweat rate, respiration rate and glucose level can be monitored. Data obtained by sensors can be used as a predictor of health status. Thus, this literature has covered the physical human activity recognition chain using wearable sensors. Initially, in order to make the data suitable for analysis, obtained sensor's data are preprocessed and segmented. Feature extraction stage is used to find a high resolution of data representation for each segment. Extracted features can be analyzed by four main methods: time domain, frequency domain, learning techniques, and other techniques. The classifier (classification algorithm/technique) uses the extracted features to differentiate between different human activities. Importantly, this review paper maybe used as a report of all algorithms used in human activity researches.

\section{REFERENCES}

[1] N. Black, D. W. Johnston, C. Propper, and M. A. Shields, "The effect of school sports facilities on physical activity, health and socioeconomic status in adulthood," Social Science \& Medicine, vol. 220, pp. 120-128, 2019.

[2] F. Atiq et al., "Sports participation and physical activity in patients with von Willebrand disease," Haemophilia, vol. 25, no. 1, pp. 101-108, 2019.

[3] K. Kapadia, H. Abdel-Jaber, F. Thabtah, and W. Hadi, "Sport analytics for cricket game results using machine learning: An experimental study," Applied Computing and Informatics, 2019.

[4] Z.-B. Cao, "Physical Activity Levels and Physical Activity Recommendations in Japan," in Physical Activity, Exercise, Sedentary Behavior and Health: Springer, 2015, pp. 3-15.

[5] K. L. Piercy et al., "The physical activity guidelines for Americans," Jama, vol. 320, no. 19, pp. 2020-2028, 2018.

[6] L. Q. Rogers, S. J. Carter, G. Williams, and K. S. Courneya, "Physical activity," in Handbook of Cancer Survivorship: Springer, 2018, pp. 287-307.

[7] M. B. Cueto-Martín, C. Márquez, E. Morales-Ortiz, and C. Pérez-Díaz, "Effect of joint physical activity on the physical condition of parents and children," 2018.

[8] J. M. Thomsen, R. B. Powell, and C. Monz, "A Systematic Review of the Physical and Mental Health Benefits of Wildland Recreation," Journal of Park \& Recreation Administration, vol. 36, no. 1, 2018.

[9] R. M. Hulteen, P. J. Morgan, L. M. Barnett, D. F. Stodden, and D. R. Lubans, "Development of foundational movement skills: A conceptual model for physical activity across the lifespan," Sports medicine, vol. 48, no. 7, pp. 1533-1540, 2018.

[10] J. E. Donnelly et al., "Physical activity, fitness, cognitive function, and academic achievement in children: a systematic review," Medicine and science in sports and exercise, vol. 48, no. 6, p. 1197, 2016.

[11] A. Afshin et al., "Information technology and lifestyle: a systematic evaluation of internet and mobile interventions for improving diet, physical activity, obesity, tobacco, and alcohol use," Journal of the American Heart Association, vol. 5, no. 9, p. e003058, 2016.

[12] I. Mocanu, O.-A. Schpor, B. Cramariuc, and L. Rusu, "Mobile@ Old: A Smart Home Platform for Enhancing the Elderly Mobility," Advances in Electrical and Computer Engineering, vol. 17, no. 4, pp. 19-27, 2017.

[13] I. Ungurean and A. Brezulianu, "An internet of things framework for remote monitoring of the healthcare parameters," Advances in Electrical and Computer Engineering, vol. 17, no. 2, pp. 11-16, 2017.

[14] R. Bailey, E. Cope, and D. Parnell, "Realising the benefits of sports and physical activity: the human capital model," RETOS. Nuevas Tendencias en Educación Física, Deporte y Recreación, no. 28, pp. 147-154, 2015.

[15] B. A. Lewis, M. A. Napolitano, M. P. Buman, D. M. Williams, and C. R. Nigg, "Future directions in physical activity intervention research: expanding our focus to sedentary behaviors, technology, and dissemination," Journal of behavioral medicine, vol. 40, no. 1, pp. 112-126, 2017.

[16] F. Kuncan, Y. KAYA, and M. Kuncan, "A Novel Approach for Activity Recognition with Down-Sampling 1D Local Binary Pattern," Advances in Electrical and Computer Engineering, vol. 19, no. 1, 2019.

[17] H. Yhdego et al., "Towards musculoskeletal simulation-aware fall injury mitigation: transfer learning with deep CNN for fall detection," in 2019 Spring Simulation Conference (SpringSim), 2019, pp. 1-12: IEEE.

[18] A. Chan, N. Ferdosi, and R. Narasimhan, "Fall detection using machine learning," ed: Google Patents, 2019.

[19] F. J. Thilo, S. Hahn, R. J. Halfens, and J. M. Schols, "Usability of a wearable fall detection prototype from the perspective of older people-A real field testing approach," Journal of clinical nursing, vol. 28, no. 1-2, pp. 310320, 2019. 
[20] O. AlShorman, B. AlShorman, M. Alkhassaweneh, F. Alkahtani, "A Review of Internet of Medical Things (IoMT) - Based Remote Health Monitoring through Wearable Sensors: A Case Study for Diabetic Patients, " Indonesian Journal of Electrical Engineering and Computer Science, vol. 20, no. 1, 2020.

[21] A. Ukil and S. Bandyopadhyay, "Automated Cardiac Health Screening Using Smartphone and Wearable Sensors Through Anomaly Analytics," in Mobile Solutions and Their Usefulness in Everyday Life: Springer, 2019, pp. $145-172$.

[22] V. Ghasemi, A. A. Pouyan, and M. Sharifi, "Human Activity Recognition in Smart Homes Based on a Difference of Convex Programming Problem," TIIS, vol. 11, no. 1, pp. 321-344, 2017.

[23] M. A. Labrador and O. D. L. Yejas, Human activity recognition: Using wearable sensors and smartphones. Chapman and Hall/CRC, 2013.

[24] J. Guo, Y. Mu, M. Xiong, Y. Liu, and J. Gu, "Activity feature solving based on TF-IDF for activity recognition in smart homes," Complexity, vol. 2019, 2019.

[25] Z. Liouane, T. Lemlouma, P. Roose, F. Weis, and H. Messaoud, "An improved extreme learning machine model for the prediction of human scenarios in smart homes," Applied Intelligence, vol. 48, no. 8, pp. 2017-2030, 2018.

[26] W. Qi, H. Su, C. Yang, G. Ferrigno, E. De Momi, and A. Aliverti, "A Fast and Robust Deep Convolutional Neural Networks for Complex Human Activity Recognition Using Smartphone," Sensors, vol. 19, no. 17, p. 3731, 2019.

[27] F. Cruciani, I. Cleland, C. Nugent, P. McCullagh, K. Synnes, and J. Hallberg, "Automatic annotation for human activity recognition in free living using a smartphone," Sensors, vol. 18, no. 7, p. 2203, 2018.

[28] X. Zheng, M. Wang, and J. Ordieres-Meré, "Comparison of Data Preprocessing Approaches for Applying Deep Learning to Human Activity Recognition in the Context of Industry 4.0," Sensors, vol. 18, no. 7, p. 2146, 2018.

[29] R. San-Segundo, J. M. Montero, R. Barra-Chicote, F. Fernández, and J. M. Pardo, "Feature extraction from smartphone inertial signals for human activity segmentation," Signal Processing, vol. 120, pp. 359-372, 2016.

[30] F. Li, K. Shirahama, M. Nisar, L. Köping, and M. Grzegorzek, "Comparison of feature learning methods for human activity recognition using wearable sensors," Sensors, vol. 18, no. 2, p. 679, 2018.

[31] N. H. Van Nguyen, M. T. Pham, N. Dai Ung, and K. Tachibana, "Human Activity Recognition Based on Weighted Sum Method and Combination of Feature Extraction Methods," International Journal of Intelligent Information Systems, vol. 7, no. 1, p. 9, 2018.

[32] K. Wnuk and N. J. Witchey, "Activity recognition systems and methods," ed: Google Patents, 2019.

[33] J. Suto, S. Oniga, and P. P. Sitar, "Feature analysis to human activity recognition," International Journal of Computers Communications \& Control, vol. 12, no. 1, pp. 116-130, 2017.

[34] O. D. Lara and M. A. Labrador, "A survey on human activity recognition using wearable sensors," IEEE communications surveys \& tutorials, vol. 15, no. 3, pp. 1192-1209, 2012.

[35] C. Chen, R. Jafari, and N. Kehtarnavaz, "A survey of depth and inertial sensor fusion for human action recognition," Multimedia Tools and Applications, vol. 76, no. 3, pp. 4405-4425, 2017.

[36] J.-L. Reyes-Ortiz, L. Oneto, A. Samà, X. Parra, and D. Anguita, "Transition-aware human activity recognition using smartphones," Neurocomputing, vol. 171, pp. 754-767, 2016.

[37] D. Yazdansepas et al., "A multi-featured approach for wearable sensor-based human activity recognition," in 2016 IEEE International Conference on Healthcare Informatics (ICHI), 2016, pp. 423-431: IEEE.

[38] Y. Chen and C. Shen, "Performance analysis of smartphone-sensor behavior for human activity recognition," Ieee Access, vol. 5, pp. 3095-3110, 2017.

[39] D. R. Faria, M. Vieira, C. Premebida, and U. Nunes, "Probabilistic human daily activity recognition towards robot-assisted living," in 2015 24th IEEE international symposium on robot and human interactive communication (RO-MAN), 2015, pp. 582-587: IEEE.

[40] K. Ali, A. X. Liu, W. Wang, and M. Shahzad, "Keystroke recognition using wifi signals," in Proceedings of the 21st Annual International Conference on Mobile Computing and Networking, 2015, pp. 90-102: ACM.

[41] A. Wang, G. Chen, J. Yang, S. Zhao, and C.-Y. Chang, "A comparative study on human activity recognition using inertial sensors in a smartphone," IEEE Sensors Journal, vol. 16, no. 11, pp. 4566-4578, 2016.

[42] J. Zhang, B. Wei, W. Hu, and S. S. Kanhere, "Wifi-id: Human identification using wifi signal," in 2016 International Conference on Distributed Computing in Sensor Systems (DCOSS), 2016, pp. 75-82: IEEE.

[43] C. Shen, Y. Chen, and G. Yang, "On motion-sensor behavior analysis for human-activity recognition via smartphones," in 2016 Ieee International Conference on Identity, Security and Behavior Analysis (Isba), 2016, pp. 1-6: IEEE.

[44] L. Cai, X. Liu, H. Ding, and F. Chen, "Human action recognition using improved sparse Gaussian process latent variable model and hidden conditional random filed," IEEE Access, vol. 6, pp. 20047-20057, 2018.

[45] S. Yousefi, H. Narui, S. Dayal, S. Ermon, and S. Valaee, "A survey on behavior recognition using wifi channel state information," IEEE Communications Magazine, vol. 55, no. 10, pp. 98-104, 2017.

[46] W. Jiang and Z. Yin, "Human activity recognition using wearable sensors by deep convolutional neural networks," in Proceedings of the 23rd ACM international conference on Multimedia, 2015, pp. 1307-1310: Acm.

[47] G. Vavoulas, C. Chatzaki, T. Malliotakis, M. Pediaditis, and M. Tsiknakis, "The MobiAct Dataset: Recognition of Activities of Daily Living using Smartphones," in ICT4AgeingWell, 2016, pp. 143-151.

[48] W. Wang, A. X. Liu, M. Shahzad, K. Ling, and S. Lu, "Device-free human activity recognition using commercial WiFi devices," IEEE Journal on Selected Areas in Communications, vol. 35, no. 5, pp. 1118-1131, 2017.

[49] L. Fan, Z. Wang, and H. Wang, "Human activity recognition model based on decision tree," in 2013 International Conference on Advanced Cloud and Big Data, 2013, pp. 64-68: IEEE. 
[50] A. Khan, N. Hammerla, S. Mellor, and T. Plötz, "Optimising sampling rates for accelerometer-based human activity recognition," Pattern Recognition Letters, vol. 73, pp. 33-40, 2016.

[51] Y. Li, D. Shi, B. Ding, and D. Liu, "Unsupervised feature learning for human activity recognition using smartphone sensors," in Mining intelligence and knowledge exploration: Springer, 2014, pp. 99-107.

[52] O. Yurur, C. H. Liu, and W. Moreno, "A survey of context-aware middleware designs for human activity recognition," IEEE Communications Magazine, vol. 52, no. 6, pp. 24-31, 2014.

[53] R. San-Segundo, H. Blunck, J. Moreno-Pimentel, A. Stisen, and M. Gil-Martín, "Robust Human Activity Recognition using smartwatches and smartphones," Engineering Applications of Artificial Intelligence, vol. 72, pp. 190-202, 2018.

[54] I. P. Machado, A. L. Gomes, H. Gamboa, V. Paixão, and R. M. Costa, "Human activity data discovery from triaxial accelerometer sensor: Non-supervised learning sensitivity to feature extraction parametrization," Information Processing \& Management, vol. 51, no. 2, pp. 204-214, 2015.

[55] S. R. Vanrell, D. H. Milone, and H. L. Rufiner, "Assessment of homomorphic analysis for human activity recognition from acceleration signals," IEEE journal of biomedical and health informatics, vol. 22, no. 4, pp. 1001-1010, 2017

[56] J. Suto and S. Oniga, "Efficiency investigation of artificial neural networks in human activity recognition," Journal of Ambient Intelligence and Humanized Computing, vol. 9, no. 4, pp. 1049-1060, 2018.

[57] J. Suto and S. Oniga, "Efficiency investigation from shallow to deep neural network techniques in human activity recognition," Cognitive Systems Research, vol. 54, pp. 37-49, 2019.

[58] P. Asghari, E. Soelimani, and E. Nazerfard, "Online Human Activity Recognition Employing Hierarchical Hidden Markov Models," arXiv preprint arXiv:1903.04820, 2019.

[59] P. Asghari and E. Nazerfard, "Activity Recognition using Hierarchical Hidden Markov Models on Streaming Sensor Data," in 2018 9th International Symposium on Telecommunications (IST), 2018, pp. 416-420: IEEE.

[60] G. Anitha and S. B. Priya, "Posture based health monitoring and unusual behavior recognition system for elderly using dynamic Bayesian network," Cluster Computing, pp. 1-8, 2018

[61] S. Siddiqui, M. A. Khan, K. Bashir, M. Sharif, F. Azam, and M. Y. Javed, "Human action recognition: a construction of codebook by discriminative features selection approach," International Journal of Applied Pattern Recognition, vol. 5, no. 3, pp. 206-228, 2018.

[62] K. Wang, J. He, and L. Zhang, "Attention-based Convolutional Neural Network for Weakly Labeled Human Activities Recognition with Wearable Sensors," IEEE Sensors Journal, 2019.

[63] J. Trelinski and B. Kwolek, "Ensemble of Classifiers Using CNN and Hand-Crafted Features for Depth-Based Action Recognition," in International Conference on Artificial Intelligence and Soft Computing, 2019, pp. 91103: Springer.

[64] N. B. Gaikwad, V. Tiwari, A. Keskar, and N. Shivaprakash, "Efficient FPGA Implementation of Multilayer Perceptron for Real-time Human Activity Classification," IEEE Access, vol. 7, pp. 26696-26706, 2019.

[65] A. M. Welhenge and A. Taparugssanagorn, "Human activity classification using long short-term memory network," Signal, Image and Video Processing, vol. 13, no. 4, pp. 651-656, 2019.

[66] X. Gao, H. Luo, Q. Wang, F. Zhao, L. Ye, and Y. Zhang, "A Human Activity Recognition Algorithm Based on Stacking Denoising Autoencoder and LightGBM," Sensors, vol. 19, no. 4, p. 947, 2019.

[67] M. Ahmad, A. Khan, M. Mazzara, and S. Distefano, "Seeking optimum system settings for physical activity recognition on smartwatches," in Science and Information Conference, 2019, pp. 220-233: Springer.

[68] M. M. Hassan, M. G. R. Alam, M. Z. Uddin, S. Huda, A. Almogren, and G. Fortino, "Human emotion recognition using deep belief network architecture," Information Fusion, vol. 51, pp. 10-18, 2019.

[69] S. Ghazal, U. Khan, M. Saleem, N. Rashid, and J. Iqbal, "Human Activity Recognition using 2D Skeleton Data and Supervised Machine Learning," IET Image Processing, 2019.

[70] S. Balli, E. A. Sağbaş, and M. Peker, "Human activity recognition from smart watch sensor data using a hybrid of principal component analysis and random forest algorithm," Measurement and Control, vol. 52, no. 1-2, pp. 3745,2019

[71] L. E. Ali, M. Z. Islam, B. Madhu, M. F. Bulbul, and N. Parveen, "Shape and Texture Features based Human Action Recognition Using Collaborative Representation Classification," 2019.

[72] D. Vishwakarma, R. Kapoor, and A. Dhiman, "Unified framework for human activity recognition: an approach using spatial edge distribution and $\mathfrak{R}$-transform," AEU-International Journal of Electronics and Communications, vol. 70, no. 3, pp. 341-353, 2016.

[73] M. F. Aslan, A. Durdu, and K. Sabanci, "Human action recognition with bag of visual words using different machine learning methods and hyperparameter optimization," Neural Computing and Applications, pp. 1-13, 2019.

[74] W. W. Myo, W. Wettayaprasit, and P. Aiyarak, "A Noble Feature Selection Method for Human Activity Recognition using Linearly Dependent Concept (LDC)," in Proceedings of the 2018 7th International Conference on Software and Computer Applications, 2018, pp. 173-177: ACM.

[75] M. F. A. bin Abdullah, A. F. P. Negara, M. S. Sayeed, D.-J. Choi, and K. S. Muthu, "Classification algorithms in human activity recognition using smartphones," International Journal of Computer and Information Engineering, vol. 6, no. 77-84, p. 106, 2012.

[76] H. F. Nweke, Y. W. Teh, M. A. Al-Garadi, and U. R. Alo, "Deep learning algorithms for human activity recognition using mobile and wearable sensor networks: State of the art and research challenges," Expert Systems with Applications, vol. 105, pp. 233-261, 2018. 
[77] J. Yin, Q. Yang, and J. J. Pan, "Sensor-based abnormal human-activity detection," IEEE Transactions on Knowledge and Data Engineering, vol. 20, no. 8, pp. 1082-1090, 2008.

[78] B. Minor and D. J. Cook, "Forecasting occurrences of activities," Pervasive and mobile computing, vol. 38, pp. 77-91, 2017.

[79] W. Liu, H. Liu, D. Tao, Y. Wang, and K. Lu, "Multiview Hessian regularized logistic regression for action recognition," Signal Processing, vol. 110, pp. 101-107, 2015.

[80] H. Rahmani, D. Q. Huynh, A. Mahmood, and A. Mian, "Discriminative human action classification using locality-constrained linear coding," Pattern recognition letters, vol. 72, pp. 62-71, 2016.

[81] D. Trabelsi, S. Mohammed, F. Chamroukhi, L. Oukhellou, and Y. Amirat, "An unsupervised approach for automatic activity recognition based on hidden Markov model regression," IEEE Transactions on automation science and engineering, vol. 10, no. 3, pp. 829-835, 2013.

[82] M. Z. Uddin, J. Lee, and T.-S. Kim, "Independent shape component-based human activity recognition via Hidden Markov Model," Applied Intelligence, vol. 33, no. 2, pp. 193-206, 2010.

[83] A. Bayat, M. Pomplun, and D. A. Tran, "A study on human activity recognition using accelerometer data from smartphones," Procedia Computer Science, vol. 34, pp. 450-457, 2014.

[84] Z. Feng, L. Mo, and M. Li, "A Random Forest-based ensemble method for activity recognition," in 2015 37th Annual International Conference of the IEEE Engineering in Medicine and Biology Society (EMBC), 2015, pp. 5074-5077: IEEE.

[85] M. Janidarmian, A. Roshan Fekr, K. Radecka, and Z. Zilic, "A comprehensive analysis on wearable acceleration sensors in human activity recognition," Sensors, vol. 17, no. 3, p. 529, 2017.

[86] T. Zebin, P. J. Scully, and K. B. Ozanyan, "Evaluation of supervised classification algorithms for human activity recognition with inertial sensors," in 2017 IEEE SENSORS, 2017, pp. 1-3: IEEE.

[87] H. Naveed, G. Khan, A. U. Khan, A. Siddiqi, and M. U. G. Khan, "Human activity recognition using mixture of heterogeneous features and sequential minimal optimization," International Journal of Machine Learning and Cybernetics, vol. 10, no. 9, pp. 2329-2340, 2019.

[88] A. Manzi, P. Dario, and F. Cavallo, "A human activity recognition system based on dynamic clustering of skeleton data," Sensors, vol. 17, no. 5, p. 1100, 2017.

[89] B. Yao, H. Hagras, M. J. Alhaddad, and D. Alghazzawi, "A fuzzy logic-based system for the automation of human behavior recognition using machine vision in intelligent environments," Soft Computing, vol. 19, no. 2, pp. 499-506, 2015.

[90] N. Wan and G. Lin, "Classifying human activity patterns from smartphone collected GPS data: A fuzzy classification and aggregation approach," Transactions in GIS, vol. 20, no. 6, pp. 869-886, 2016.

[91] M. O. Omisore, O. W. Samuel, and E. J. Atajeromavwo, "A Genetic-Neuro-Fuzzy inferential model for diagnosis of tuberculosis," Applied Computing and Informatics, vol. 13, no. 1, pp. 27-37, 2017.

[92] W. He, Y. Guo, and K. C. Yow, "Recognition of human activities using a multiclass relevance vector machine," Optical Engineering, vol. 51, no. 1, p. 017202, 2012.

[93] A. Oikonomopoulos, M. Pantic, and I. Patras, "Sparse B-spline polynomial descriptors for human activity recognition," Image and vision computing, vol. 27, no. 12, pp. 1814-1825, 2009.

[94] I. Aydin, "Fuzzy integral and cuckoo search based classifier fusion for human action recognition," Advances in Electrical and Computer Engineering, vol. 18, no. 1, pp. 3-11, 2018.

[95] R. Khemchandani and S. Sharma, "Robust least squares twin support vector machine for human activity recognition," Applied Soft Computing, vol. 47, pp. 33-46, 2016.

[96] D. N. Tran and D. D. Phan, "Human activities recognition in android smartphone using support vector machine," in 2016 7th International Conference on Intelligent Systems, Modelling and Simulation (ISMS), 2016, pp. 64-68: IEEE.

[97] Y. Zheng, "Human activity recognition based on the hierarchical feature selection and classification framework," Journal of Electrical and Computer Engineering, vol. 2015, p. 34, 2015.

[98] Y. Chen, M. Guo, and Z. Wang, "An improved algorithm for human activity recognition using wearable sensors," in 2016 Eighth International Conference on Advanced Computational Intelligence (ICACI), 2016, pp. 248-252: IEEE.

[99] S. Shariat and V. Pavlovic, "A new adaptive segmental matching measure for human activity recognition," in Proceedings of the IEEE International Conference on Computer Vision, 2013, pp. 3583-3590.

[100] Y. Dedeoğlu, B. U. Töreyin, U. Güdükbay, and A. E. Çetin, "Silhouette-based method for object classification and human action recognition in video," in European Conference on Computer Vision, 2006, pp. 64-77: Springer.

[101] L. Piyathilaka and S. Kodagoda, "Gaussian mixture based HMM for human daily activity recognition using 3D skeleton features," in 2013 IEEE 8th conference on industrial electronics and applications (ICIEA), 2013, pp. 567-572: IEEE.

[102] D. D. Dawn and S. H. Shaikh, "A comprehensive survey of human action recognition with spatio-temporal interest point (STIP) detector," The Visual Computer, vol. 32, no. 3, pp. 289-306, 2016.

[103] M. Shoaib, S. Bosch, O. Incel, H. Scholten, and P. Havinga, "Complex human activity recognition using smartphone and wrist-worn motion sensors," Sensors, vol. 16, no. 4, p. 426, 2016.

[104] G. Chetty, M. White, and F. Akther, "Smart phone based data mining for human activity recognition," Procedia Computer Science, vol. 46, pp. 1181-1187, 2015. 
[105] W. Wang, A. D. Degenhart, G. P. Sudre, D. A. Pomerleau, and E. C. Tyler-Kabara, "Decoding semantic information from human electrocorticographic (ECoG) signals," in 2011 Annual International Conference of the IEEE Engineering in Medicine and Biology Society, 2011, pp. 6294-6298: IEEE.

[106] X. Zou and B. Bhanu, "Anomalous activity classification in the distributed camera network," in 2008 15th IEEE International Conference on Image Processing, 2008, pp. 781-784: IEEE.

[107] H. J. Escalante, E. F. Morales, and L. E. Sucar, "A naive bayes baseline for early gesture recognition," Pattern Recognition Letters, vol. 73, pp. 91-99, 2016.

[108] D. Moreira-Arce, P. M. Vergara, and S. Boutin, "Diurnal human activity and introduced species affect occurrence of carnivores in a human-dominated landscape," PLoS one, vol. 10, no. 9, p. e0137854, 2015.

[109] T. L. van Kasteren, G. Englebienne, and B. J. Kröse, "Human activity recognition from wireless sensor network data: Benchmark and software," in Activity recognition in pervasive intelligent environments: Springer, 2011, pp. 165-186.

[110] F. Chamroukhi, S. Mohammed, D. Trabelsi, L. Oukhellou, and Y. Amirat, "Joint segmentation of multivariate time series with hidden process regression for human activity recognition," Neurocomputing, vol. 120, pp. 633644, 2013.

[111] L. Piyathilaka and S. Kodagoda, "Human activity recognition for domestic robots," in Field and Service Robotics, 2015, pp. 395-408: Springer.

[112] A. Nazabal, P. Garcia-Moreno, A. Artes-Rodriguez, and Z. Ghahramani, "Human activity recognition by combining a small number of classifiers," IEEE journal of biomedical and health informatics, vol. 20, no. 5, pp. 1342-1351, 2015.

[113] A. Subasi, M. Radhwan, R. Kurdi, and K. Khateeb, "IoT based mobile healthcare system for human activity recognition," in 2018 15th Learning and Technology Conference (L\&T), 2018, pp. 29-34: IEEE.

[114] A. D. Ignatov and V. V. Strijov, "Human activity recognition using quasiperiodic time series collected from a single tri-axial accelerometer," Multimedia tools and applications, vol. 75, no. 12, pp. 7257-7270, 2016.

[115] F. J. Ordóñez, J. A. Iglesias, P. De Toledo, A. Ledezma, and A. Sanchis, "Online activity recognition using evolving classifiers," Expert Systems with Applications, vol. 40, no. 4, pp. 1248-1255, 2013.

[116] H. Kerdegari, K. Samsudin, A. R. Ramli, and S. Mokaram, "Evaluation of fall detection classification approaches," in 2012 4th International Conference on Intelligent and Advanced Systems (ICIAS2012), 2012, vol. 1, pp. 131-136: IEEE.

[117] M. A. Ayu, S. A. Ismail, A. F. A. Matin, and T. Mantoro, "A comparison study of classifier algorithms for mobile-phone's accelerometer based activity recognition," Procedia Engineering, vol. 41, pp. 224-229, 2012.

[118] F. Attal, S. Mohammed, M. Dedabrishvili, F. Chamroukhi, L. Oukhellou, and Y. Amirat, "Physical human activity recognition using wearable sensors," Sensors, vol. 15, no. 12, pp. 31314-31338, 2015.

[119] L. Sharma, D. K. Yadav, and A. Singh, "Fisher's linear discriminant ratio based threshold for moving human detection in thermal video," Infrared Physics \& Technology, vol. 78, pp. 118-128, 2016.

[120] Q. Zhu, Z. Chen, and Y. C. Soh, "Smartphone-based human activity recognition in buildings using localityconstrained linear coding," in 2015 IEEE 10th Conference on Industrial Electronics and Applications (ICIEA), 2015, pp. 214-219: IEEE.

[121] N. Zerrouki, F. Harrou, Y. Sun, and A. Houacine, "Vision-based human action classification using adaptive boosting algorithm," IEEE Sensors Journal, vol. 18, no. 12, pp. 5115-5121, 2018.

[122] P. Li, Y. Wang, Y. Tian, T.-S. Zhou, and J.-s. Li, "An automatic user-adapted physical activity classification method using smartphones," IEEE Transactions on Biomedical Engineering, vol. 64, no. 3, pp. 706-714, 2016.

[123] J. Ye, G. Qi, N. Zhuang, H. Hu, and K. A. Hua, "Learning compact features for human activity recognition via probabilistic first-take-all," IEEE transactions on pattern analysis and machine intelligence, 2018.

[124] B. Zhou, J. Yang, and Q. Li, "Smartphone-Based Activity Recognition for Indoor Localization Using a Convolutional Neural Network," Sensors, vol. 19, no. 3, p. 621, 2019.

[125] P. P. Markopoulos and F. Ahmad, "Robust radar-based human motion recognition with L1-norm linear discriminant analysis," in 2018 IEEE International Microwave Biomedical Conference (IMBioC), 2018, pp. 145147: IEEE.

[126] H. H. Ali, H. M. Moftah, and A. A. Youssif, "Depth-based human activity recognition: A comparative perspective study on feature extraction," Future Computing and Informatics Journal, vol. 3, no. 1, pp. 51-67, 2018.

[127] A. Ahmadi, M. Behroozi, V. Shalchyan, and M. R. Daliri, "Classification of epileptic EEG signals by wavelet based CFC," in 2018 Electric Electronics, Computer Science, Biomedical Engineerings' Meeting (EBBT), 2018, pp. 1-4: IEEE.

[128] P. Siirtola, H. Koskimäki, and J. Röning, "OpenHAR: A Matlab Toolbox for Easy Access to Publicly Open Human Activity Data Sets-Introduction and Experimental Results," in Human Activity Sensing: Springer, 2019, pp. 121-133.

[129] S. Scheurer, S. Tedesco, K. N. Brown, and B. O'Flynn, "Human activity recognition for emergency first responders via body-worn inertial sensors," in 2017 IEEE 14th International Conference on Wearable and Implantable Body Sensor Networks (BSN), 2017, pp. 5-8: IEEE.

[130] K. Gusain, A. Gupta, and B. Popli, "Transition-aware human activity recognition using extreme gradient boosted decision trees," in Advanced Computing and Communication Technologies: Springer, 2018, pp. 41-49.

[131] W. Gao, C. Ruan, and R. Xu, "Sensor-based semantic-level human activity recognition using temporal classification," Technical report, Stanford University2016. 
[132] A.-A. Liu, W.-Z. Nie, Y.-T. Su, L. Ma, T. Hao, and Z.-X. Yang, "Coupled hidden conditional random fields for RGB-D human action recognition," Signal Processing, vol. 112, pp. 74-82, 2015.

[133] M. H. Siddiqi, R. Ali, A. M. Khan, Y.-T. Park, and S. Lee, "Human facial expression recognition using stepwise linear discriminant analysis and hidden conditional random fields," IEEE Transactions on Image Processing, vol. 24, no. 4, pp. 1386-1398, 2015.

[134] R. DiPietro et al., "Recognizing surgical activities with recurrent neural networks," in International conference on medical image computing and computer-assisted intervention, 2016, pp. 551-558: Springer.

[135] J. Ye, G. Stevenson, and S. Dobson, "KCAR: A knowledge-driven approach for concurrent activity recognition," Pervasive and Mobile Computing, vol. 19, pp. 47-70, 2015.

[136] L. Ballan, M. Bertini, A. Del Bimbo, L. Seidenari, and G. Serra, "Effective codebooks for human action representation and classification in unconstrained videos," IEEE Transactions on Multimedia, vol. 14, no. 4, pp. 1234-1245, 2012.

[137] S. Bhattacharya, P. Nurmi, N. Hammerla, and T. Plötz, "Using unlabeled data in a sparse-coding framework for human activity recognition," Pervasive and Mobile Computing, vol. 15, pp. 242-262, 2014.

[138] R. J. Javier and Y. Kim, "Application of linear predictive coding for human activity classification based on micro-Doppler signatures," IEEE Geoscience and Remote Sensing Letters, vol. 11, no. 10, pp. 1831-1834, 2014.

[139] Y. Kim and T. Moon, "Human detection and activity classification based on micro-Doppler signatures using deep convolutional neural networks," IEEE geoscience and remote sensing letters, vol. 13, no. 1, pp. 8-12, 2015.

[140] V. B. Semwal, M. Raj, and G. C. Nandi, "Biometric gait identification based on a multilayer perceptron," Robotics and Autonomous Systems, vol. 65, pp. 65-75, 2015.

[141] K. H. Walse, R. V. Dharaskar, and V. M. Thakare, "Pca based optimal ann classifiers for human activity recognition using mobile sensors data," in Proceedings of First International Conference on Information and Communication Technology for Intelligent Systems: Volume 1, 2016, pp. 429-436: Springer.

[142] J. Yang, M. N. Nguyen, P. P. San, X. L. Li, and S. Krishnaswamy, "Deep convolutional neural networks on multichannel time series for human activity recognition," in Twenty-Fourth International Joint Conference on Artificial Intelligence, 2015.

[143] K. Altun, B. Barshan, and O. Tunçel, "Comparative study on classifying human activities with miniature inertial and magnetic sensors," Pattern Recognition, vol. 43, no. 10, pp. 3605-3620, 2010.

[144] R. Madarshahian, J. M. Caicedo, and N. Haerens, "Human Activity Benchmark Classification Using Multilayer Artificial Neural Network," in Dynamics of Civil Structures, Volume 2: Springer, 2019, pp. 207-210.

[145] M. A. Khan, T. Akram, M. Sharif, M. Y. Javed, N. Muhammad, and M. Yasmin, "An implementation of optimized framework for action classification using multilayers neural network on selected fused features," Pattern Analysis and Applications, vol. 22, no. 4, pp. 1377-1397, 2019.

[146] M. Chen, Y. Li, X. Luo, W. Wang, L. Wang, and W. Zhao, "A novel human activity recognition scheme for smart health using multilayer extreme learning machine," IEEE Internet of Things Journal, vol. 6, no. 2, pp. $1410-1418,2018$.

[147] C. A. Ronao and S.-B. Cho, "Human activity recognition with smartphone sensors using deep learning neural networks," Expert systems with applications, vol. 59, pp. 235-244, 2016.

[148] J. Park, R. Javier, T. Moon, and Y. Kim, "Micro-Doppler based classification of human aquatic activities via transfer learning of convolutional neural networks," Sensors, vol. 16, no. 12, p. 1990, 2016.

[149] A. Ignatov, "Real-time human activity recognition from accelerometer data using Convolutional Neural Networks," Applied Soft Computing, vol. 62, pp. 915-922, 2018.

[150] Y. Kim and Y. Li, "Human activity classification with transmission and reflection coefficients of on-body antennas through deep convolutional neural networks," IEEE Transactions on Antennas and Propagation, vol. 65 , no. 5, pp. 2764-2768, 2017.

[151] J. C. Nunez, R. Cabido, J. J. Pantrigo, A. S. Montemayor, and J. F. Velez, "Convolutional neural networks and long short-term memory for skeleton-based human activity and hand gesture recognition," Pattern Recognition, vol. 76, pp. 80-94, 2018.

[152] S. Matsui, N. Inoue, Y. Akagi, G. Nagino, and K. Shinoda, "User adaptation of convolutional neural network for human activity recognition," in 2017 25th European Signal Processing Conference (EUSIPCO), 2017, pp. $753-$ 757: IEEE.

[153] S. Ha and S. Choi, "Convolutional neural networks for human activity recognition using multiple accelerometer and gyroscope sensors," in 2016 International Joint Conference on Neural Networks (IJCNN), 2016, pp. 381388: IEEE.

[154] M. Z. Uddin, M. M. Hassan, A. Almogren, A. Alamri, M. Alrubaian, and G. Fortino, "Facial expression recognition utilizing local direction-based robust features and deep belief network," IEEE Access, vol. 5, pp. 4525-4536, 2017.

[155] J. Zhang, Y. Wu, J. Bai, and F. Chen, "Automatic sleep stage classification based on sparse deep belief net and combination of multiple classifiers," Transactions of the Institute of Measurement and Control, vol. 38, no. 4, pp. 435-451, 2016.

[156] Y. Rizk, N. Hajj, N. Mitri, and M. Awad, "Deep belief networks and cortical algorithms: A comparative study for supervised classification," Applied Computing and Informatics, 2018.

[157] L. Wang, "Recognition of human activities using continuous autoencoders with wearable sensors," Sensors, vol. 16, no. 2, p. $189,2016$. 
[158] M. S. Seyfioğlu, A. M. Özbayoğlu, and S. Z. Gürbüz, "Deep convolutional autoencoder for radar-based classification of similar aided and unaided human activities," IEEE Transactions on Aerospace and Electronic Systems, vol. 54, no. 4, pp. 1709-1723, 2018.

[159] H. Zou, Y. Zhou, J. Yang, H. Jiang, L. Xie, and C. J. Spanos, "Deepsense: Device-free human activity recognition via autoencoder long-term recurrent convolutional network," in 2018 IEEE International Conference on Communications (ICC), 2018, pp. 1-6: IEEE.

[160] F. Gu, K. Khoshelham, S. Valaee, J. Shang, and R. Zhang, "Locomotion activity recognition using stacked denoising autoencoders," IEEE Internet of Things Journal, vol. 5, no. 3, pp. 2085-2093, 2018.

[161] M. Gnouma, A. Ladjailia, R. Ejbali, and M. Zaied, "Stacked sparse autoencoder and history of binary motion image for human activity recognition," Multimedia Tools and Applications, vol. 78, no. 2, pp. 2157-2179, 2019.

[162] S. Liu, L. Kong, and H. Wang, "Human Activities Recognition Based on Skeleton Information via Sparse Representation," Journal of Computing Science and Engineering, vol. 12, no. 1, pp. 1-11, 2018.

[163] W. Liu, Z.-J. Zha, Y. Wang, K. Lu, and D. Tao, "\$ p \$-Laplacian regularized sparse coding for human activity recognition," IEEE Transactions on Industrial Electronics, vol. 63, no. 8, pp. 5120-5129, 2016.

[164] W. Wang, Y. Yan, L. Zhang, R. Hong, and N. Sebe, "Collaborative sparse coding for multiview action recognition," IEEE MultiMedia, vol. 23, no. 4, pp. 80-87, 2016.

[165] A. Murad and J.-Y. Pyun, "Deep recurrent neural networks for human activity recognition," Sensors, vol. 17, no. 11, p. 2556, 2017.

[166] M. Inoue, S. Inoue, and T. Nishida, "Deep recurrent neural network for mobile human activity recognition with high throughput," Artificial Life and Robotics, vol. 23, no. 2, pp. 173-185, 2018.

[167] F. Ordóñez and D. Roggen, "Deep convolutional and lstm recurrent neural networks for multimodal wearable activity recognition," Sensors, vol. 16, no. 1, p. 115, 2016.

[168] N. Y. Hammerla, S. Halloran, and T. Plötz, "Deep, convolutional, and recurrent models for human activity recognition using wearables," arXiv preprint arXiv:1604.08880, 2016.

[169] L. Wang, "Three-dimensional convolutional restricted Boltzmann machine for human behavior recognition from RGB-D video," EURASIP Journal on Image and Video Processing, vol. 2018, no. 1, p. 120, 2018.

[170] D. C. Mocanu et al., "Factored four way conditional restricted boltzmann machines for activity recognition," Pattern Recognition Letters, vol. 66, pp. 100-108, 2015.

[171] D. C. Mocanu, H. B. Ammar, L. Puig, E. Eaton, and A. Liotta, "Estimating 3d trajectories from 2d projections via disjunctive factored four-way conditional restricted boltzmann machines," Pattern Recognition, vol. 69, pp. 325$335,2017$.

[172] P. Lei and S. Todorovic, "Modeling human-skeleton motion patterns using conditional deep Boltzmann machine," in 2016 23rd International Conference on Pattern Recognition (ICPR), 2016, pp. 1845-1850: IEEE.

[173] Z. Chen, L. Zhang, Z. Cao, and J. Guo, "Distilling the knowledge from handcrafted features for human activity recognition," IEEE Transactions on Industrial Informatics, vol. 14, no. 10, pp. 4334-4342, 2018

[174] G. De Leonardis et al., "Human Activity Recognition by Wearable Sensors: Comparison of different classifiers for real-time applications," in 2018 IEEE International Symposium on Medical Measurements and Applications $(M e M e A), 2018$, pp. 1-6: IEEE.

[175] K. Nakano and B. Chakraborty, "Effect of dynamic feature for human activity recognition using smartphone sensors," in 2017 IEEE 8th International Conference on Awareness Science and Technology (iCAST), 2017, pp. 539-543: IEEE.

[176] A. Kamišalić, I. Fister, M. Turkanović, and S. Karakatič, "Sensors and functionalities of non-invasive wristwearable devices: A review," Sensors, vol. 18, no. 6, p. 1714, 2018.

[177] M. Bariya, H. Y. Y. Nyein, and A. Javey, "Wearable sweat sensors," Nature Electronics, vol. 1, no. 3, p. 160, 2018.

[178] M. Bariya et al., "Roll-to-Roll Gravure Printed Electrochemical Sensors for Wearable and Medical Devices," ACS nano, vol. 12, no. 7, pp. 6978-6987, 2018.

[179] J. Kim, A. S. Campbell, and J. Wang, "Wearable non-invasive epidermal glucose sensors: A review," Talanta, vol. 177, pp. 163-170, 2018.

[180] M. Dehghani, A. M. Abubakar, and M. Pashna, "Market-driven management of start-ups: The case of wearable technology," Applied Computing and Informatics, 2018.

[181] J. Matthews, K. T. Win, H. Oinas-Kukkonen, and M. Freeman, "Persuasive technology in mobile applications promoting physical activity: a systematic review," Journal of medical systems, vol. 40, no. 3, p. 72, 2016.

[182] M. A. Case, H. A. Burwick, K. G. Volpp, and M. S. Patel, "Accuracy of smartphone applications and wearable devices for tracking physical activity data," Jama, vol. 313, no. 6, pp. 625-626, 2015.

[183] S. Chatterjee, A. K. Das, and J. K. Sing, "A novel and efficient user access control scheme for wireless body area sensor networks," Journal of King Saud University-Computer and Information Sciences, vol. 26, no. 2, pp. 181201, 2014.

[184] O. AlShorman, B. Alshorman, F. Alkahtani, "A review of wearable sensors based monitoring with daily physical activity to manage type 2 diabetes, "International Journal of Electrical and Computer Engineering (IJECE), vol.11, no. 1, 2021.

[185] S. Malwade et al., "Mobile and wearable technologies in healthcare for the ageing population," Computer methods and programs in biomedicine, vol. 161, pp. 233-237, 2018.

[186] H. Yildirim and A. M. Ali-Eldin, "A model for predicting user intention to use wearable IoT devices at the workplace," Journal of King Saud University-Computer and Information Sciences, vol. 31, no. 4, pp. 497-505, 2019. 
[187] A. Birenboim, M. Dijst, F. E. Scheepers, M. P. Poelman, and M. Helbich, "Wearables and location tracking technologies for mental-state sensing in outdoor environments," The Professional Geographer, pp. 1-13, 2019.

[188] S. M. Phillips, L. Cadmus-Bertram, D. Rosenberg, M. P. Buman, and B. M. Lynch, "Wearable technology and physical activity in chronic disease: opportunities and challenges," American journal of preventive medicine, vol. 54, no. 1, pp. 144-150, 2018.

[189] K. Bagot et al., "Current, future and potential use of mobile and wearable technologies and social media data in the ABCD study to increase understanding of contributors to child health," Developmental cognitive neuroscience, vol. 32, pp. 121-129, 2018.

[190] A. Godfrey, V. Hetherington, H. Shum, P. Bonato, N. Lovell, and S. Stuart, "From A to Z: Wearable technology explained," Maturitas, vol. 113, pp. 40-47, 2018.

[191] T. N. Gia et al., "Energy efficient wearable sensor node for IoT-based fall detection systems," Microprocessors and Microsystems, vol. 56, pp. 34-46, 2018.

[192] L. Chen and C. D. Nugent, "Sensor-Based Activity Recognition Review," in Human Activity Recognition and Behaviour Analysis: Springer, 2019, pp. 23-47.

[193] G. Civitarese, C. Bettini, T. Sztyler, D. Riboni, and H. Stuckenschmidt, "newNECTAR: Collaborative active learning for knowledge-based probabilistic activity recognition," Pervasive and Mobile Computing, vol. 56, pp. 88-105, 2019.

[194] G. Bleser, B. Taetz, and P. Lukowicz, "Human Motion Capturing and Activity Recognition Using Wearable Sensor Networks," in Developing Support Technologies: Springer, 2018, pp. 191-206.

[195] M. Vrigkas, C. Nikou, and I. A. Kakadiaris, "A review of human activity recognition methods," Frontiers in Robotics and AI, vol. 2, p. 28, 2015.

[196] N. F. Butte et al., "A youth compendium of physical activities: activity codes and metabolic intensities," Medicine and science in sports and exercise, vol. 50, no. 2, p. 246, 2018.

[197] M. Ziaeefard and R. Bergevin, "Semantic human activity recognition: A literature review," Pattern Recognition, vol. 48, no. 8, pp. 2329-2345, 2015.

[198] M. Ermes, J. Pärkkä, J. Mäntyjärvi, and I. Korhonen, "Detection of daily activities and sports with wearable sensors in controlled and uncontrolled conditions," IEEE transactions on information technology in biomedicine, vol. 12 , no. 1, pp. 20-26, 2008.

[199] S. C. Mukhopadhyay, "Wearable sensors for human activity monitoring: A review," IEEE sensors journal, vol. 15, no. 3, pp. 1321-1330, 2014.

[200] A. Baldominos, Y. Saez, and P. Isasi, "Feature set optimization for physical activity recognition using genetic algorithms," in Proceedings of the Companion Publication of the 2015 Annual Conference on Genetic and Evolutionary Computation, 2015, pp. 1311-1318: ACM.

[201] W. Gao, H. Ota, D. Kiriya, K. Takei, and A. Javey, "Flexible electronics toward wearable sensing," Accounts of chemical research, vol. 52, no. 3, pp. 523-533, 2019.

[202] S. C. Mukhopadhyay, Wearable electronics sensors: For safe and healthy living. Springer, 2015.

[203] J. Lee, K. Wheeler, and D. A. James, Wearable Sensors in Sport: A Practical Guide to Usage and Implementation. Springer, 2019.

[204] C. Massaroni et al., "Smart textile based on piezoresistive sensing elements for respiratory monitoring," IEEE Sensors Journal, 2019.

[205] A. Brueck, T. Iftekhar, A. Stannard, K. Yelamarthi, and T. Kaya, "A real-time wireless sweat rate measurement system for physical activity monitoring," Sensors, vol. 18, no. 2, p. 533, 2018.

[206] L. Fiorini et al., "Foot inertial sensing for combined cognitive-motor exercise of the sustained attention domain," IEEE Transactions on Biomedical Engineering, vol. 66, no. 8, pp. 2413-2420, 2019.

[207] M. L. S. de Asteasu, N. Martínez-Velilla, F. Zambom-Ferraresi, Á. Casas-Herrero, N. Millor, and M. Izquierdo, "Quantifying physical functional trajectory in hospitalized older adults using body worn inertial sensors," Journal of biomechanics, 2019.

[208] H. Wennman et al., "Gender, age and socioeconomic variation in 24-hour physical activity by wrist-worn accelerometers: the FinHealth 2017 Survey," Scientific reports, vol. 9, no. 1, p. 6534, 2019.

[209] T. W. Markison, S. Kiaei, and G. McCoy, "Wireless in-shoe physical activity monitoring apparatus," ed: Google Patents, 2018

[210] P. Kokkinos, P. Narayan, and A. Pittaras, "Physical Activity, Blood Pressure, and Cardiac Structure," Cardiorespiratory Fitness in Cardiometabolic Diseases: Prevention and Management in Clinical Practice, p. $181,2019$.

[211] W. Chen, "Thermometry and interpretation of body temperature," Biomedical engineering letters, vol. 9, no. 1, pp. 3-17, 2019.

[212] V. Martínez-Vizcaíno et al., "Effectiveness of a school-based physical activity intervention on adiposity, fitness and blood pressure: MOVI-KIDS study," Br J Sports Med, pp. bjsports-2018-099655, 2019.

[213] D. Margarito, E. González, I. Buendía, M. Selman, and M. Hautefeuille, "Development of a wearable for oximetry and patient physical activity correlation," in Smart Photonic and Optoelectronic Integrated Circuits XX, 2018, vol. 10536, p. 1053622: International Society for Optics and Photonics.

[214] S. Tagougui, N. Taleb, and R. Rabasa-Lhoret, "The benefits and limits of technological advances in glucose management around physical activity in patients type 1 diabetes," Frontiers in endocrinology, vol. 9, 2018.

[215] A. Romeo et al., "Can Smartphone Apps Increase Physical Activity? Systematic Review and Meta-Analysis," Journal of medical Internet research, vol. 21, no. 3, p. e12053, 2019. 
[216] M. V. McConnell, M. P. Turakhia, R. A. Harrington, A. C. King, and E. A. Ashley, "Mobile health advances in physical activity, fitness, and atrial fibrillation: moving hearts," Journal of the American College of Cardiology, vol. 71, no. 23, pp. 2691-2701, 2018.

[217] W. Niu, J. Long, D. Han, and Y.-F. Wang, "Human activity detection and recognition for video surveillance," in 2004 IEEE International Conference on Multimedia and Expo (ICME)(IEEE Cat. No. 04TH8763), 2004, vol. 1, pp. 719-722: IEEE.

[218] H. Martin, D. Bucher, E. Suel, P. Zhao, F. Perez-Cruz, and M. Raubal, "Graph Convolutional Neural Networks for Human Activity Purpose Imputation from GPS-based Trajectory Data," 2018.

[219] A. I. Ramzi and M. A.-L. El-Bedawi, "Towards integration of remote sensing and GIS to manage primary health care centers," Applied Computing and Informatics, vol. 15, no. 2, pp. 109-113, 2019.

[220] J. Qi, P. Yang, A. Waraich, Z. Deng, Y. Zhao, and Y. Yang, "Examining sensor-based physical activity recognition and monitoring for healthcare using Internet of Things: A systematic review," Journal of biomedical informatics, vol. 87, pp. 138-153, 2018.

[221] O. AlShorman, T. Ali, and M. Irfan, "EEG Analysis for Pre-learning Stress in the Brain," in Asian Simulation Conference, 2017, pp. 447-455: Springer.

[222] O. M. AlShorman and A. M. Alshorman, "Frontal lobe and long-term memory retrieval analysis during prelearning stress using EEG signals," Bulletin of Electrical Engineering and Informatics, vol. 9, no. 1, 2020.

[223] N. Inomata, W. Suwa, N. Van Toan, M. Toda, and T. Ono, "Resonant magnetic sensor using concentration of magnetic field gradient by asymmetric permalloy plates," Microsystem Technologies, vol. 25, no. 10, pp. 39833989, 2019.

[224] Y. Fu, Human activity recognition and prediction. Springer, 2016.

[225] Y. E. Ustev, O. Durmaz Incel, and C. Ersoy, "User, device and orientation independent human activity recognition on mobile phones: Challenges and a proposal," in Proceedings of the 2013 ACM conference on Pervasive and ubiquitous computing adjunct publication, 2013, pp. 1427-1436: ACM.

[226] A. Hosseini et al., "Children Activity Recognition: Challenges and Strategies," in 2018 40th Annual International Conference of the IEEE Engineering in Medicine and Biology Society (EMBC), 2018, pp. 4331-4334: IEEE.

[227] J. T. Sunny, S. M. George, J. J. Kizhakkethottam, J. T. Sunny, S. M. George, and J. J. Kizhakkethottam, "Applications and challenges of human activity recognition using sensors in a smart environment," IJIRST Int. J. Innov. Res. Sci. Technol, vol. 2, pp. 50-57, 2015.

[228] R. Gravina, P. Alinia, H. Ghasemzadeh, and G. Fortino, "Multi-sensor fusion in body sensor networks: State-ofthe-art and research challenges," Information Fusion, vol. 35, pp. 68-80, 2017.

[229] M. Dimiccoli, A. Cartas, and P. Radeva, "Activity recognition from visual lifelogs: state of the art and future challenges," in Multimodal Behavior Analysis in the Wild: Elsevier, 2019, pp. 121-134.

[230] A. D. Antar, M. Ahmed, and M. A. R. Ahad, "Challenges in Sensor-based Human Activity Recognition and a Comparative Analysis of Benchmark Datasets: A Review," in 2019 Joint 8th International Conference on Informatics, Electronics \& Vision (ICIEV) and 2019 3rd International Conference on Imaging, Vision \& Pattern Recognition (icIVPR), 2019, pp. 134-139: IEEE.

[231] M. Awais, S. Mellone, and L. Chiari, "Physical activity classification meets daily life: Review on existing methodologies and open challenges," in 2015 37th Annual International Conference of the IEEE Engineering in Medicine and Biology Society (EMBC), 2015, pp. 5050-5053: IEEE.

[232] P. Lameski, A. Dimitrievski, E. Zdravevski, V. Trajkovik, and S. Koceski, "Challenges in data collection in realworld environments for activity recognition," in IEEE EUROCON 2019-18th International Conference on Smart Technologies, 2019, pp. 1-5: IEEE.

[233] M. Alzahrani and S. Kammoun, "Human Activity Recognition: Challenges and Process Stages," International Journal of Innovative Research in Computer and Communication Engineering, vol. 5, pp. 1111-1118, 2016.

[234] A. Bulling, U. Blanke, and B. Schiele, "A tutorial on human activity recognition using body-worn inertial sensors," ACM Computing Surveys (CSUR), vol. 46, no. 3, p. 33, 2014.

[235] A. A. A. Ari, O. K. Ngangmo, C. Titouna, O. Thiare, A. Mohamadou, and A. M. Gueroui, "Enabling Privacy and Security in Cloud of Things: architecture, applications, security \& privacy challenges," Applied Computing and Informatics, 2019.

[236] P. P. Ray, "A survey on Internet of Things architectures," Journal of King Saud University-Computer and Information Sciences, vol. 30, no. 3, pp. 291-319, 2018.

[237] V. Palanisamy and R. Thirunavukarasu, "Implications of big data analytics in developing healthcare frameworksA review," Journal of King Saud University-Computer and Information Sciences, vol. 31, no. 4, pp. 415-425, 2019.

[238] M. Al-khassaweneh and O. AlShorman, "Frei-Chen Bases Based Lossy Digital Image Compression Technique," Applied Computing and Informatics, 2020

[239] A. Secerbegovic, A. Gogic, N. Suljanovic, M. Zajc, and A. Mujcic, "Computational Balancing between Wearable Sensor and Smartphone towards Energy-Efficient Remote Healthcare Monitoring," Advances in Electrical and Computer Engineering, vol. 18, no. 4, pp. 3-11, 2018. 